\title{
The discussion on the combination of Number and Shape in the heuristic teaching of higher mathematics
}

\author{
Wenhao Xie \\ School of Mathematical Sciences \\ Xi'an Shiyou University \\ ShaanXi, Xi'an \\ 1609632928@qq.com \\ Jin jin Liang \\ School of Mathematical Sciences \\ Xi'an Shiyou University \\ ShaanXi, Xi'an \\ myonlyonly@126.com
}

\author{
Xiao qun Sun \\ School of Mathematical Sciences \\ Xi'an Shiyou University \\ ShaanXi, Xi'an \\ 409802000@qq.com \\ Xiaoyan Wang \\ School of Mathematical Sciences \\ Xi'an Shiyou University \\ ShaanXi, Xi'an \\ shiyouwxy@126.com
}

\begin{abstract}
Number and shape are the important research objects in the mathematical research. The method of combination of Number and Shape make the number and shape to produce the connection. Heuristic teaching is a kind of teaching thought that can guide students think positively step by step and learn actively according to the characteristics of the teaching content and students' knowledge and thinking level. This paper discusses the practical significance about the combination of Number and Shape in the heuristic teaching of higher mathematics. Through specific case analysis, we recognize that the use of this teaching method can make students more deeply understand the connection inside the higher mathematics knowledge, and improve the enthusiasm of students to participate in classroom teaching activities.
\end{abstract}

Keywords-The combination of Number and Shape; Heuristic teaching; Higher mathematics.

\section{INTRODUCTION}

Heuristic teaching refers to a kind of guiding thought rather than a kind of specific supervising method. The discussion about heuristic teaching of 《Higher mathematics》, neither has the fixed teaching modes, also has no fixed teaching method. Specifically, the teaching method which reflects fundamental characteristics of the heuristic teaching, can be referred to as the method of heuristic of teaching[1].

Heuristic teaching needs that the teacher guides the students to obtain knowledge through their own efforts by certain appropriate teaching methods. The aim is regarding the students as the main body of teaching activity, inspires their active thinking, and cultivates their learning initiative and enthusiasm. The heuristic teaching can inspire the students' intrinsic motivation of the knowledge acquisition and stimulate the students' desire of learning and discovery science.
The combination of number and shape is a kind of mathematical thinking method; it combines the abstract mathematical language, quantity relationship with intuitional geometric figure and location relationship. It can make complex problem simply and abstract problems specifically by the combination of abstract thinking and imaginal thinking. Thus, it can achieve the aim of optimization solving problem ways. The combination of number and shape includes two aspects of "explaination shape with number" and "helping number with shape".

\section{THE SIGNIFICANCE OF THE COMBINATION OF NUMBER AND SHAPE IN THE HEURISTIC TEACHING OF HIGHER MATHEMATICS}

The function of the combination of the number and shape teaching thought in the heriustic teaching [2]:

- It is helpful for the students to understand deeply the mathematical concept; it makes the comprehension of the mathematical concepts perfectly and harmoniously for the students. This comprehension and the process of the knowledge detailing is the process that guides the students to actively explore, it is not blindly "indoctrination".

- The application of the combination of number and shape in heuristic teaching is helpful for the students to deepen mathematical thinking and solve the mathematical problems with multi-perspective and multi-methods. And this kind of training about mathematical thinking can develop and improve the students' learning ability and the ability of using knowledge.

- The application of the combination of number and shape in heuristic teaching is helpful for the students to develop the ability of intuition thinking and deepen the 
comprehension of the essence of the mathematical problems

- It can help the students feeling the beauty of mathematics.

\section{THE APPLICATION OF THE COMBINATION OF NUMBER AND} SHAPE IN THE HEURISTIC TEACHING OF HIGHER MATHEMATICS

A. In class guidance, if we can properly introduce the teaching method of the combination of the number and shape, the students can easily enter the teaching situation, so as to inspire the students consciously and actively thinking, naturally accepting the new knowledge.

Example1: In the section of the "definite integral definition", before the proposed question of the calculation on the area of the "curved trapezoid", we can arrange the teaching links like this: we first draw three graphics:1.rectangle (figure1) ;2. We change one side of the rectangle to a tiny continuous curve radian ( figure2);3. We will change the tiny continuous curve radian of figure 2 to a continuous and vary in a large range curve (figure 3 ), and then let the students calculate their areas. The area of figure 1 is the most easy to calculate because the area of the rectangle equals to the length multiplication by the height. For figure 2, as a result of the height change of the arc is very small; we use the high of a point on the hem approximately instead of the other points, that is to say, using the ideas of replacing the curve with straight line and replacing the area of the curved trapezoid with the area of the rectangle. Of course, the change of "curve high" of the curved trapezoid is smaller; the accuracy of the approximate replacement is higher. When calculating the area of the figure 3 , because the change of the "curve" is larger, so if we use directly the method of figure 2 to replace approximately, the error will be larger. How do we inspire the students to use the ideas of replacing the curve with straight line in the case of getting small errors? Students will think of using the methods of figure 2. Then we will further inspire the students how to do reference to the situation of figure 2? Possibly some students will put forward that the bottom margin can be devided a lot of small zones, due to the curve is continuous, so in a very small range, the change of the curve is tiny, we can use the method of figure 2 to calculate the approximate area of each small divisiory curved trapezoid, then we can calculate the approximate area of the big curved trapezoid by summation. Thus we can introduce naturally this method of "segmentation, approximate calculation, summation, takes the limit", finally we get the area of the curved trapezoid, which leads to the definition of definite integral.

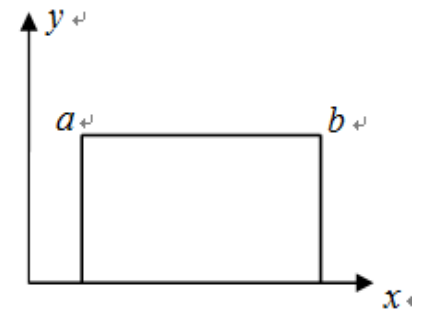

Fig. 1. Rectangle

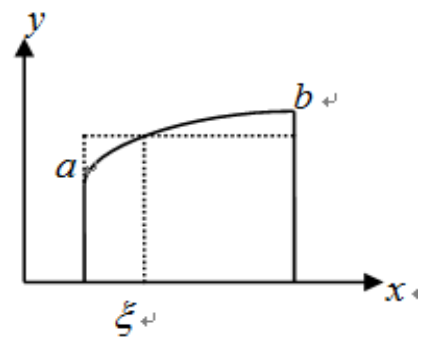

Fig. 2. Curved trapezoid with a tiny change continuous curve

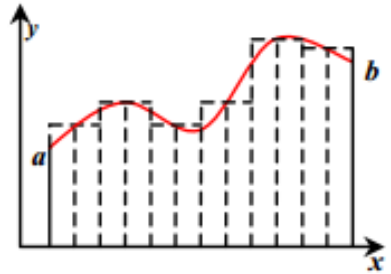

Fig. 3. Curved trapezoid with a large change continuous curve

B. In the process of teaching, in order to making the students to deepen their understanding on some important definitions and theorems, if the teachers can well design the teaching methods combination with the graphics to guide the students into the teaching steps, they can help the students better understand the definitions and theorems.It will obtain the unexpected teaching effects.

Example2 : In the section of "differential mean value theorem", we should special emphasis on the three conditions of the "rolle's theorem" after proving the "rolle's theorem": 1 . Be continuous on the closed interval;2. Be derivable on the open interval;3. $f(a)=f(b)$. In order to let the students deepen the understanding of the conditions of this theorem, we should draw the following three functions graphics (figure 4figure 6), they respectively can't meet the needs of the first, second and third conditions of the "rolle's theorem". In the three cases, we can easily see the conclusion of the "rolle's theorem" is not established from the graphics. Without the tedious proof, we can make the students to easily remember the conditions of the theorem combination with the three special function graphics.

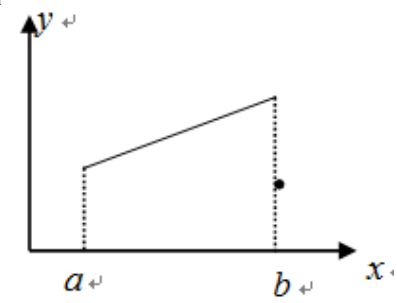

Fig. 4. Dosen't meet the first condition of the "rolle's theorem"

This paper is supported by high level college construction of XI'an shiyou University. 


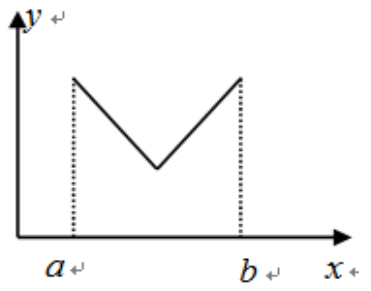

Fig. 5. Dosen't meet the second condition of the "rolle's theorem"

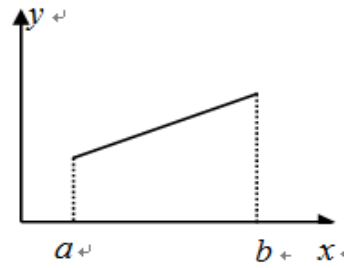

Fig. 6. Dosen't meet the third condition of the "rolle's theorem"

C. In the transition process from the old knowledge to the new knowledge, if the teachers can subtly use the graphics, it not only can inspire the students to explore the new knowledge, but also let the students make a deep impression on the learned knowlege.

Example3:In the section of "differential mean value theorem", from the "rolle's theorem" to the "Lagrange's mean value theorem", we explain the geometric meaning of the "rolle's theorem" for the students : If a function which is continuous on the closed interval and derivable on the open interval has the equal values on the interval endpoints, that is, the line between the interval endpoins is horizontal, so the conclusion of the "rolle's theorem" tells us: at least we can find one point between the interval where the tangent line of the curve parallels to the chord $\mathrm{AB}$ (Figure 7). Then, we can let the students to think about such a problem: if we freely choose two endpoints of a chord of the curve such as A and $\mathrm{B}$, that is to say, the chord is not always level. Can we accordingly promote the conclusion of the "rolle's theorem "? That is, for any chord, can we find one point in the open interval where the tangent line of the curve parallels to the chord AB? Then we will draw the graphics, not only can guide the students to actively think, and also can find the problems we proposed to be correct from the graphics(Figure 8). This is the conclusion of the "Lagrange's theorem". Thus, the "Lagrange's theorem" is derived from the "rolle's theorem". In fact, we promote the condition of the "rolle's theorem", in other words, the "rolle's theorem" is the special case of the "Lagrange's theorem". The introduction like this, not only can realize the natural transition of the new knowledge and old knowledge but also can let the students deepen the understanding of the relationship between the two theorems.

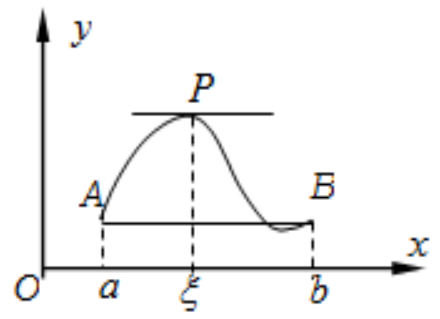

Fig .7. The "rolle's theorem"

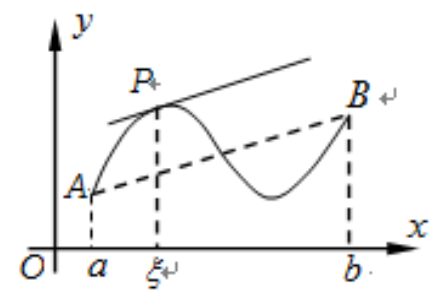

Fig.8. The "Lagrange's theorem"

\section{CONCLUSION}

Through the front cases analysis, if the teachers can correctly and properly apply the methods of the combination of number and shape into the heuristic teaching of 《Higher mathematics $\rangle$, it will largely improve the enthusiasm of the students' learning higher mathematics, cultivate their ability of actively learning, so as to grasp the connection between the knowledge points. It is an effective way to improve the students' ability. It will play a positive and important role for promoting the teaching reform and improving the quality of teaching [3].

\section{ACKNOWLEDGMENT}

Thanks to the reviewer's hard work. Thanks to the funding and support of Dean's office of Xi'an Shiyou University and the college of science of Xi'an Shiyou University.

\section{REFERENCES}

[1] Deng Zhi-ping. "The Analysis of 《Higher Mathematics》 heuristic teaching", Heilongjiang province higher education research, pp. 58-59, June 1995.

[2] Li Na-na. "The discuss about the teaching method of the combination of Number and Shape ", Journal of Inner Mongolia Normal University, vol. 26, pp.141-142, April 2013.

[3] Li Bao-ping. "The application of Heuristic teaching in the teaching of 《Higher Mathematic》》", Higher education window, pp.156, March 2010 . 